
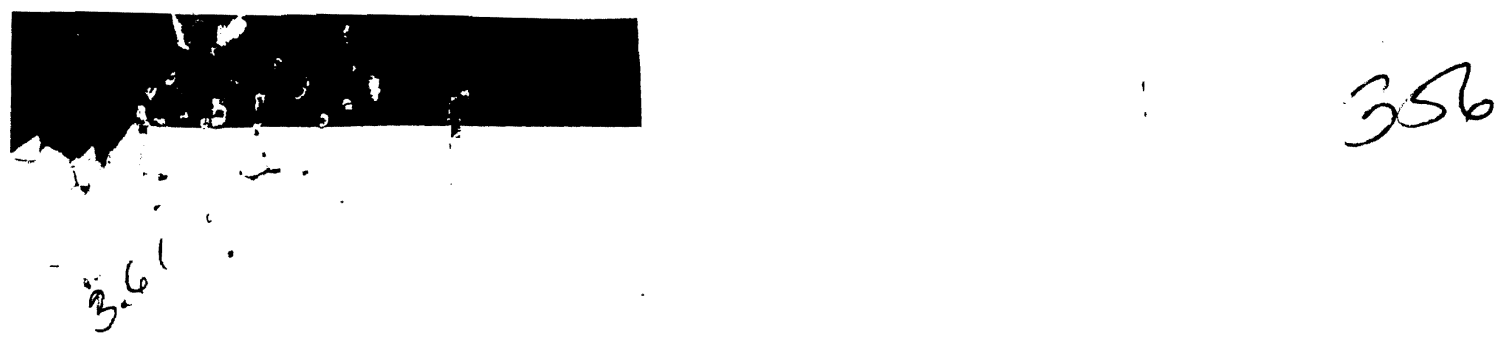

\title{
HEPATIC TRISEGMENTECTOMY AND OTHER LIVER
}

\section{RESECTIONS}

\author{
Thomas E. Starzl, M.D., F.A.C.S., Richard H. Bell, M.D., Robert W. Beart, M.D., and \\ Charles W. Putnam, M.D., Denver, Colorado
}

THE FIRST to successfully remove all of the liver located to the right of the falciform ligament, or about 80 to 85 per cent of the organ, were Wangensteen (19), Lortat-Jacob and Robert (11) and Quattlebaum (17). The potential enthusiasm for the procedure which became known as extended right hepatic lobectomy was apparently dampened by a heavy mortality rate in the early trials of its use by Brunschwig (1) and by others. Although Pack and Molander (16) and Miller (14) acquired experience with more than 40 patients in the ensuing two decades, large collections were not reported by others. More recent series of hepatic resections have contained few extended right lobectomies. For example, Longmire and his associates (10) had only two in their experience with 75 hepatic resections. There were no such operations among the 107 hepatic resections recently reported by $\operatorname{Lin}(8)$.

The literature concerning extended right hepatic lobectomy has added little about the surgical technique since the original descriptions. It is our purpose to focus upon a neglected aspect of the operation which concerns the anatomy encountered in dissecting the left branches of the portal triad toward the falciform ligament and umbilical fissure. At the same time, other principles of extended right lobectomy will be reviewed; their relevance to less extensive partial hepatectomies will be mentioned.

SURGIGAL UNITS

The basis for orderly subtotal resection of the liver is found in anatomic studies which were published a quarter of a century apart. The first of these influential reports was by McIndoe and Counseller (13) in 1927. They proved that the division between the true right and left lobes of the liver was not at the falciform ligament as had been previously believed but rather at a line going

From the Department of Surgery, University of Colorado Medical Center, and the Derver Veterans Administration Hospital, Denver.

The work was supported by research grants from the Veterans Administration; by the National Institutes of Health Grant Nos. AI-AM-08898 and AM-07772, and by the General Clinical Research Centers Program of the Division of Research Resources, search Centers Program of the Division of Research Resources,
National Institutes of Health, Grant Nos. RR-00069 and RR-00051. through the bed of the gallbladder and projecting posteriorly toward the vena cava. From 1950 to 1954, Hortsjö (7), Healey (5) and Healey and Schroy (6) demonstrated that each true lobe was further divided into two segments. The portal venous, hepatic arterial and hepatic duct branches conformed to the four segment organization. These observations were confirmed and extended by Couinaud (2) and Goldsmith and Woodburne (4). The larger hepatic veins were distributed differently since a number of their ramifications are between rather than within segments and lobes.

For practical purposes, there are only four surgical units that lend themselves to controlled excision (Fig. 1) including the right and left true lobes which consist of two segments each. The third possibility is removal of the complete right lobe plus the medial segment of the left lobe, The operation is most correctly called trisegmentectomy, although the term of Pack and his associates (15) of extended right hepatic lobectomy has been frequently used. Fourth, the liver to the left of the

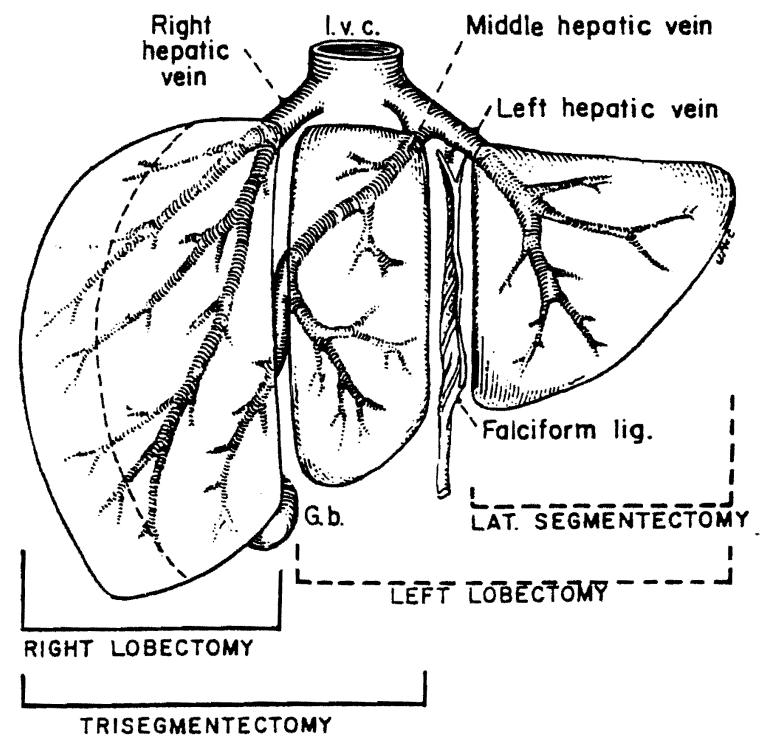

Fig. 1. The usual kinds of partial hepatectomy. Note that there are only four common resections. 
$t$

2 Surgery, Gynecology \& Obstetrics - September 1975 . Volume 141

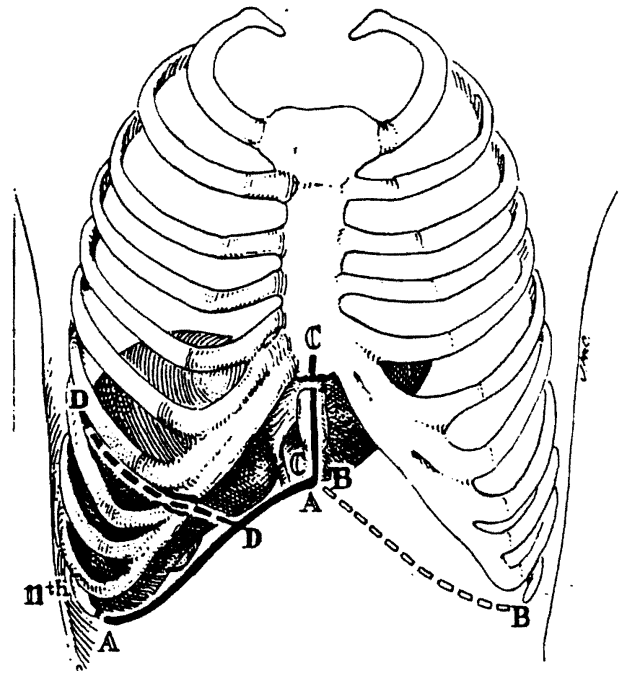

Fig. 2. Approaches for trisegmentectomy or true right lobectomy. Note that several extensions may be made from the basic right subcostal incision, $A$ to $A$, that is always used. More than one of the depicted extensions may be required in a given patient. For left hepatic lobectomy or lateral segmentectomy, mirror images of the shown extensions can be added to the basic left subcostal incision, although it is almost never necessary to have a left thoracic component.

falciform ligament is a single segment, and its removal should be known as lateral segmentectomy instead of left lobectomy.

Although resections of other single or multiple segments or subsegments are theoretically possible and actually have been carried out by $\mathrm{McBride}$ and Wallace (12), by Miller (14) and by others, we have not performed such procedures. It would be difficult, for example, to justify the isolated removal of the medial segment of the left lobe or the anterior or posterior segment of the right lobe when full lobectomy is so safe and easy.

Since 1963, 14 trisegmentectomies, five right lobectomies, five left lobectomies and three lateral segmentectomies have been performed. Eight of the patients were children one-and one-half to 16 years old, mean 9.3 years. The 19 adults were 19 to 71 years old, mean 39 years. In 25 of the 27 patients, the indication for operation was a hepatic mass. The exceptional two patients had traumatic injuries of the liver. Four of the lobectomies were performed urgently to control hemorrhage, in two caused by trauma and in the two others caused by ruptured hepatic adenomas. Nine of the 25 tumors were benign. The 16 malignant tumors were primary in the liver in 11 instances and in the gallbladder in one. Neoplasms were metastatic to the liver in one example each from the rectum, colon, kidney or right adrenal gland. The tumor of the adrenal gland was a neuroblastoma that had di- rectly invaded the liver. The malignant condition of renal origin was a Wilm's tumor, for which a left nephrectomy had previously been performed. No patient had gross cirrhosis in the liver remnant.

\section{INCISION}

A right subcostal incision is always used, of ten with extensions as shown in Figure 2. Good additional exposure may be obtained by continuing superiorly in the midline, particularly if the xiphoid process is removed. The incision may be carried across into the left subcostal area. If a thoracic extension is decided upon, the incision used by Quattlebaum (17) (Fig. 2) permits a better view of the suprahepatic inferior vena cava and right hepatic vein, and it allows the triangular and coronary ligaments to be incised under direct vision.

Most authors have considered a thoracoabdominal incision to be obligatory for right hepatic lobectomy or trisegmentectomy. Our experience has been that a thoracic component is almost never needed in children under the age of 12 years and that it is usually not required even in adults. For this reason, a thoracic extension is not made unless the need becomes evident during the early steps of dissection described subsequently. Even if a thoracic extension is made, we carry the incision in the diaphragm only half way from its periphery to the vena cava rather than completely to the vena cava. as has usually been advocated.

After exposing the liver and ruling out the possibility of extrahepatic metastases, a decision is made about the size of the resection, and a plan is developed for the steps of dissection. The sequence of the operation may vary from patient to patient depending upon the location and size of the lesion. Usually, the right triangular and coronary ligaments are incised, and the bare area is broadly entered. By so doing, it becomes possible to elevate the right lobe into the wound and to retract it toward the left when this maneuver becomes desirable at later times. Inability to mobilize the liver safely at this time or difficulty in seeing the entrance of the right hepatic vein into the inferior vena cava is the main reason to consider a thoracic extension. Having settled upon the final incision and having completed preliminary right lobar mobilization, attention is turned to the hilus. During all of these maneuvers, the falciform ligament and ligamentum teres are usually not cut, and if possible, they are retained throughout the operation.

\section{DISSECTION OF THE HIIUS}

The necessary first step in trisegmentectomy is ligation and division of the cystic artery and duct 


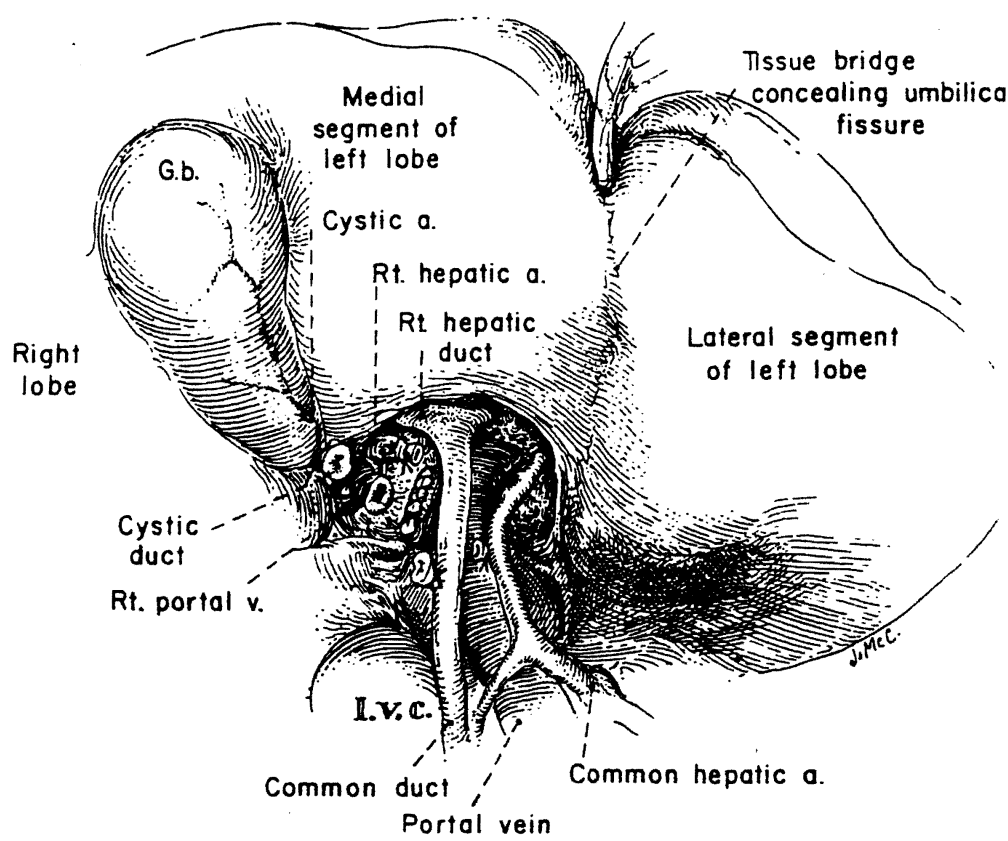

FIG. 3. Devascularization of the true right lobe. The cystic artery and cystic duct are ligated and divided to aid in the dissection. Of the structures of the portal triad, the bifurcation of the duct is almost always the most superior, that of the portal vein is intermediate and that of the hepatic artery is most inferior. The lateral suture closure of the portal vein is at the site of detachment of the right portal branch. The tissue bridge conceals the umbilical fissure, behind which a finger can be inserted. The bridge is present in about half the patients.

(Fig. 3). The cystic artery normally arises from the right hepatic artery (Fig. 3). The right branches of the structures of the portal triad are now isolated.

The right hepatic artery is sacrificed first (Fig. 3 ). The dissection for trisegmentectomy is so greatly facilitated by prior knowledge of the arterial blood supply of the liver that elective resection is never performed without preoperative aortography or celiac arteriography. In performing this examination, it is especially important to be certain of the origin of the arterial supply to the lateral segment of the left lobe, which is to be retained. Normally, the right hepatic artery originates from the proper hepatic artery (Fig. 3)-and passes posterior to the common duct. However, arterial anomalies are so numerous that ligation should netr be performed without preliminary test occlusion or without being. sure that during this occlusion there are pulsations distally in the region of the umbilical fissure.

At a more superior level, the right branch of the portal vein is detached, and the defect is closed with continuous No. 6-0 arterial suture (Fig. 3). If any difficulty is encountered, the portal vein can easily be approached posteriorly, providing the right lobe of the liver is retracted anteriorly and to the left (Fig. 4). The posterior approach is especially helpful if vein branches emanate from the back of the portal bifurcation, as is of ten the case (Fig. 4). After dividing the right hepatic artery and the right portal vein, a line of demarcation between the right and left true lobes, passing through the bed of the gallbladder and directed toward the vena cava as described in the corrosion cast studies of McIndoe and Counseller (13), is evident.

Invariably, the most superior bifurcation is the common duct (Fig. 3), sometimes within the substance of the liver. The right duct is ligated and divided where it comes off almost like a crossbar from a $\mathrm{T}$.

\section{DISSECTION OF THE LEFT BRANCHES}

Before beginning this phase of the operation, the exact location of the umbilical fissure must be determined. In many human livers, the umbilical fissure is concealed by a fusion of the inferior lips of the medial and lateral segments of the left lobe (Figs. 3 and 5). Even if the fusion is complete and involves a large surface, it can readily be opened with finger fracture (Figs. 5 and 6) since no large structures pass through it. In the base of the opened field lie the distal portions of the portal triad structures (Fig. 6).

The left branches of the triad structures which are several centimeters in length are dissected 


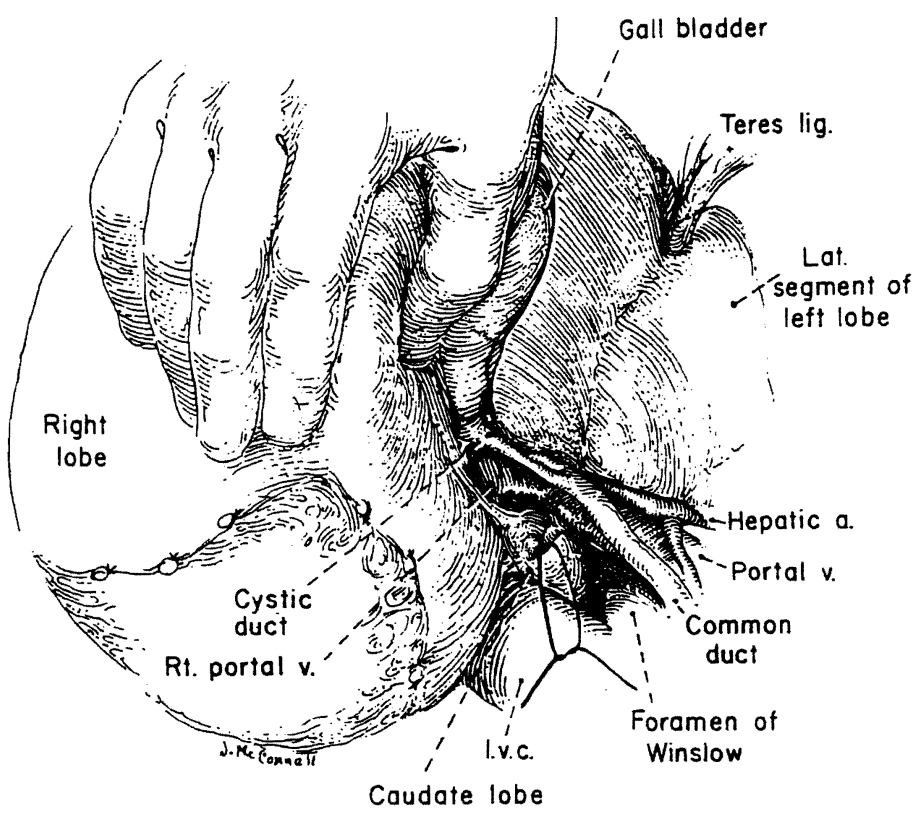

Fig. 4. Posterior approach in dissecting the bifurcation of the portal vein. This maneuver is made possible by retracting the right lobe of the liver anteriorly and to the left.

completely free from the undersurface of the liver (Fig. 5). This portion of the left portal vein is called the pars transversa, and it usually gives off several small branches to adjacent liver tissue. These fine ramifications are doubly ligated and divided. Small ducts and arteries which parallel the venous branches must be dealt with. If there is an anomalous artery to the entire left lobe or to the lateral segment arising from the celiac axis or from the left gastric artery, such an aberrant vessel almost always enters the base of the umbilical fissure considerably to the left of the field of dissection of the pars transversa and the left hepatic duct. This anomaly, if recognized, is a technical advantage since its presence virtually eliminates the dangęr of dearterializing the lateral segmental remnant:

The leftward mobilization of the left portal structures is stopped short of the umbilical fissure for the reasons to be discussed in the section on transection of the liver. The last two major branches before the fissure is reached pass posteriorly to the left portion of the caudate lobe. If a decision is made to sacrifice these branches (Fig. 5), the caudate lobe will be devascularized and ideally then should be removed in its entirety from the surface of the inferior vena cava and from its junction with the lateral segment. Such total caudate lobe removal will usually leave the lateral segment drained by a single vein, the left hepatic vein. If the final posterior branches of the left triad structures going to the caudate lobe are retained, as we believe is the usual practice in trisegmentectomy, the intrinsic risk of the operation is undoubtedly reduced. However, in several of our patients, total caudate extirpation has been necessary to obtain complete tumor removal.

\section{HEPATIC VEINS}

At this point or in some instances at an earlier stage of the operation, the right hepatic vein is encircled by cautious dissection as the right lobe is retracted. The vein is doubly clamped with angled pediatric Pott's clamps, divided and sewed shut on both sides with continuous vascular suture (Fig. 7). This maneuver is potentially dangerous because the hepatic vein is extremely short and because a tear during the dissection would create a defect in the side of the vena cava that would be difficult to control or repair. A number of more inferior, smaller hepatic veins entering the anterior surface of the retrohepatic inferior vena cava are also ligated and divided. As noted earlier, all vena caval tributaries except the left hepatic vein are ligated and divided if the caudate lobe is to be totally excised.

\section{THE LIVER TRANSECTION}

The crucial step in trisegmentectomy is the identification of the complex of venous, arterial 


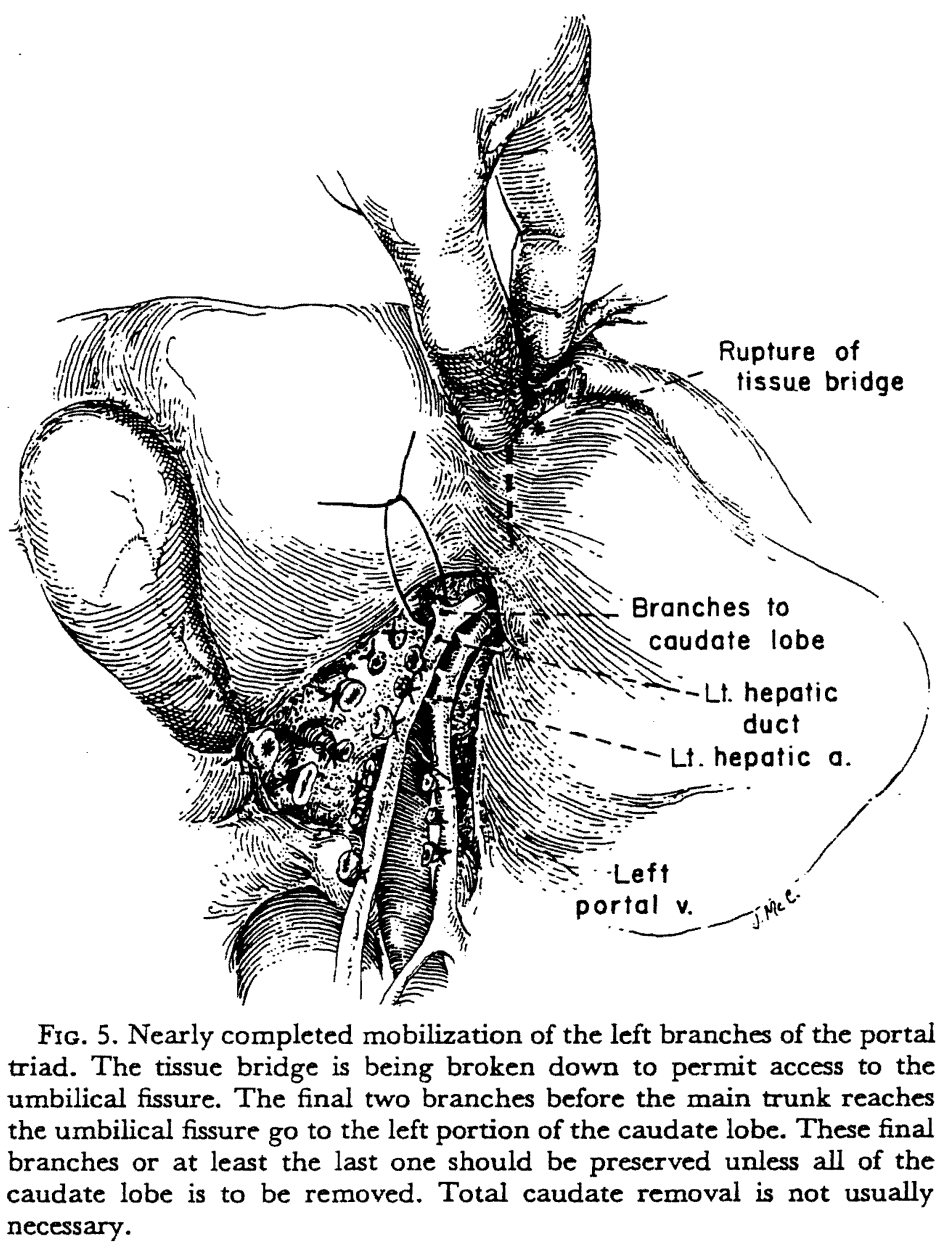

and duct structures that originate in the umbilical fissure and feed back from the main left trunks to the medial segment of the left lobe. Although these originate in the umbilical fissure, they are not dissected there. The feed-back structures are found just to the right of the falciform ligament within the substance of the liver (Fig. 6). The parenchyma of the liver can be crushed down to them, either with clamps and ligatures or with fingers (Fig. 6A). Only with the occlusion of the contained vessels does the medial segment becomeryanotic.

The feed-back blood supply and duct drainage of the medial segment were not evident in the pioneer studies of Healey (5) and Healey and Schroy (6). However, this feature was accurately noted by Goldsmith and Woodburne (4), who cautioned in connection with lateral segmentectomy that dissection in the umbilical fissure should be avoided for fear of injuring both vessels and ducts passing to the retained medial segment. Cognizance has not been taken of their warning by subsequent authors.

With all three segments devascularized, the liver is split toward the diaphragm at the exact line of color demarcation (Fig. 8). We do not develop a parasegmental line of transection leaving a centimeter or so of devitalized tissue as has been commonly advocated. Knife handles, clamps and ligatures can be used to crush down to intersegmental strands, as described by Quattlebaum (17), or else the finger fracture method of $\mathrm{Lin}$ and his associates (9) may be preferable. If the line of transection is in the appropriate place, good-sized intersegmental veins are soon encountered. These are pushed toward the lateral segment, preserved when possible and used as a guide to further cleavage. Near the diaphragm, the middle hepatic vein (Figs. 1 and 8 ) is ligated. It either enters separately into the inferior vena cava or more commonly joins the left hepatic vein to form a short common trunk (Figs. 1 and 8). The liver transection is facilitated by grasping the edges of the hepatic fragments and pulling them down (Fig. 8) and by pushing down the right lobe with the flat 


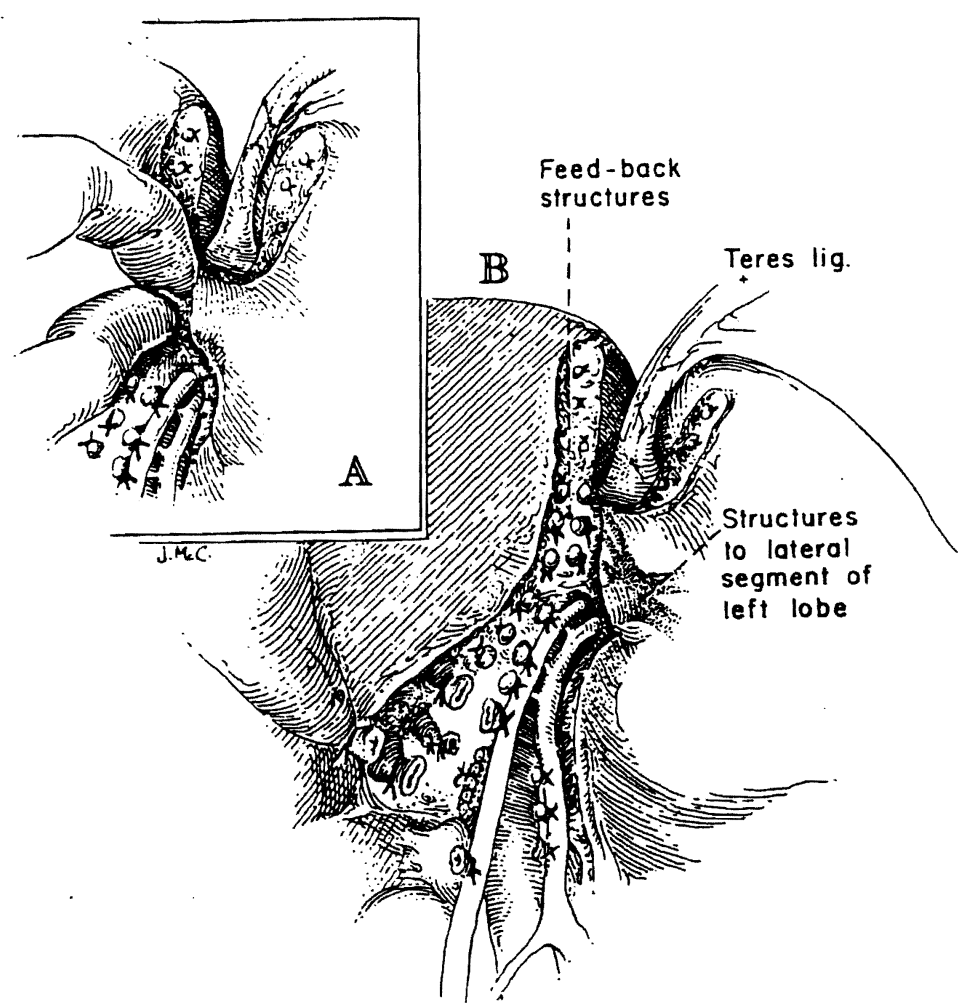

Frg. 6. Structures feeding back from the umbilical fissure to the medial segment of the left lobe. A, These are encircled usually by blunt dissection within liver substances just to the right of the falciform ligament and umbilical fissure without entering the fissure. Note that the hepatic tissue bridge concealing the umbilical fissure has been broken down. $\mathrm{B}$, The three segments of the specimen are now devascularized.

of the operator's left hand which is placed in the subphrenic space on the diaphragmatic surface of the liver. As the liver is split, anesthesia should be deep enough to prevent spontaneous respiratory efforts. If the diaphragm is intact, the negative intrathoracic pressure with breathing could result in air embolus.

After removal of the specimen, the raw surface of the residual lateral segment is pink and well vascularized. It usually is remarkably dry. Since residual bleeders are not covered over by dead tissue, they easily can be identified and suture ligated. Large intersegmental veins which are visible at the plane of transection are protected during the check for hemostasis. No effort is made to cover the raw surface with living tissue or with prosthetic materials, such as Gelfoam ${ }^{\otimes}$ (absorbable gelatine sponge) or Surgicel ${ }^{\otimes}$ (oxidized cellulose).

\section{IMPORTANCE OF DRAINAGE AND OTHER CARE}

$T$ tubes are not used. However, the huge right subphrenic dead space must be perfectly drained.
To achieve this, a portion of the wound 3 to 5 inches in length is usually left open (Fig. 9). Five to ten 1 inch drains are brought out through the wound. Gauze strip impregnated with antibiotics is packed into the wound more superficially to prevent evisceration through the large drain area. This is removed after 48 to 72 hours, and twice daily irrigations of the cavity are begun. Within a day or so, the drains also are removed. As regeneration occurs the cavity rapidly reduces in size. Within two or three weeks, the patient can be discharged with instructions for self-irrigation of the sinus tract. After four to eight weeks, all wounds are totally healed.

Other aspects of postoperative care are beyond the scope of this article, but in essence they are supportive. Antibiotics are started before operation and continued for several days after. Human albumin is frequently given for a few days postoperatively. All patients with trisegmentectomy become jaundiced, as described by Pack and Molander (16), but this finding begins to recede in a week or ten days. It has been unusual for 


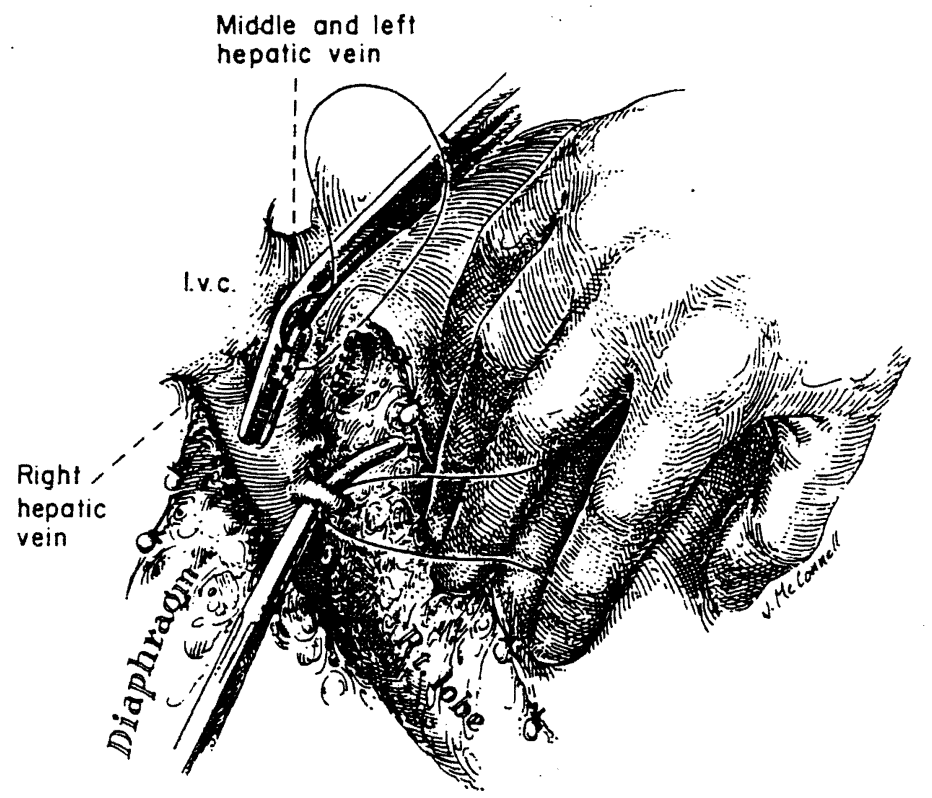

Frg. 7. Division of the right hepatic vein. With the right lobe of the liver retracted anteriorly and to the left, the vein is divided between Pott's clamps and oversewn with vascular sutures. Several smaller hepatic veins must be ligated as they enter the retrohepatic vena cava more inferiorly.

the patient not to be able to resume a diet within three or four days.

\section{RELATION TO OTHER HEPATIC RESECTIONS}

The same technical principles with appropriate modifications are applied to the other three common kinds of hepatic resection. When true right or left lobectomy is performed, the gallbladder must be removed, but the tedious dissection of the left branches of the structures of the portal triad is not necessary. As with trisegmentectomy, dead tissue is not deliberately left by the development of a parasegmental plane. However, an attempt is made to leave the middle hepatic vein with the remnant since it drains both the right and left lobes. The raw area with true lobectomy is much greater than after trisegmentectomy, but the intrinsic risk of operation is unquestionably less.

In the performance of lateral segmentectomy, the blood supply and duct drainage should be sacrificed well to the left of the falciform ligament and umbilical fissure, as was originally emphasized by Goldsmith and Woodburne (4) (Fig. 10), to avoid damaging the structures feeding back to the medial segment.

\section{MORTALITY AND MORBIDITY}

In 14 trisegmentectomies, five right lobectomies, five left lobectomies and three lateral segmentec- tomies carried out since 1963 , there has been no operative mortality. The death most proximate to operation occurred four and one-half months after trisegmentectomy for a squamous cell carcinoma that had developed in an intrahepatic cyst. The cause of death was widespread local and distant metastatic disease. Other deaths from recurrent tumor were at seven, 27 and 58 months. Eleven patients with malignant disease are living free of tumor from a few weeks to five years postoperatively. All the patients with benign disease are well from a few months to many years later.

No patients required reoperation for postoperative hemorrhage. One subphrenic abscess developed and was drained in a child in whom drainage after trisegmentectomy was inadequate. Although bile-stained fluid was occasionally irrigated in the cavities, persistent bile leakage was found in one patient; this stopped spontaneously.

\section{RECENT TECHNICAL INNOVATIONS}

Lin $(8,9)$ has advocated hepatic resection by several techniques which do not require preliminary dissection and control of the vascular and duct structures entering the proposed specimen, nor is early control obtained of the hepatic veins. The mortality rate with these techniques has been low, the operating times have been short and the blood loss has been small, particularly with 


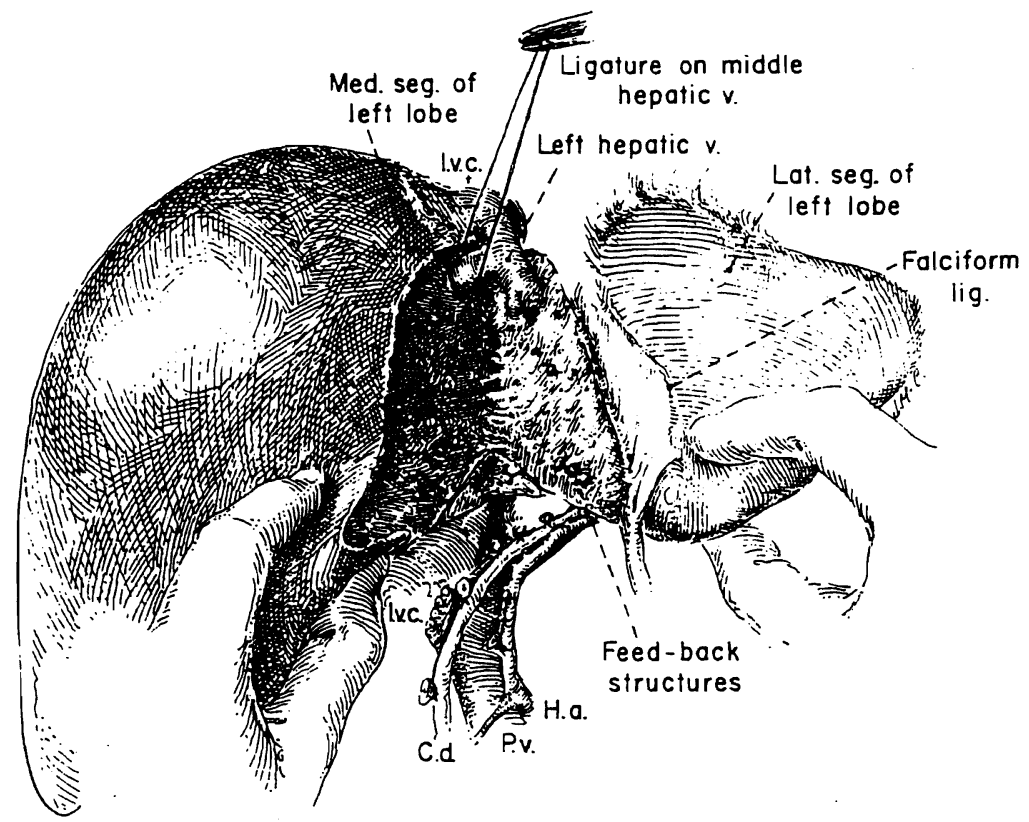

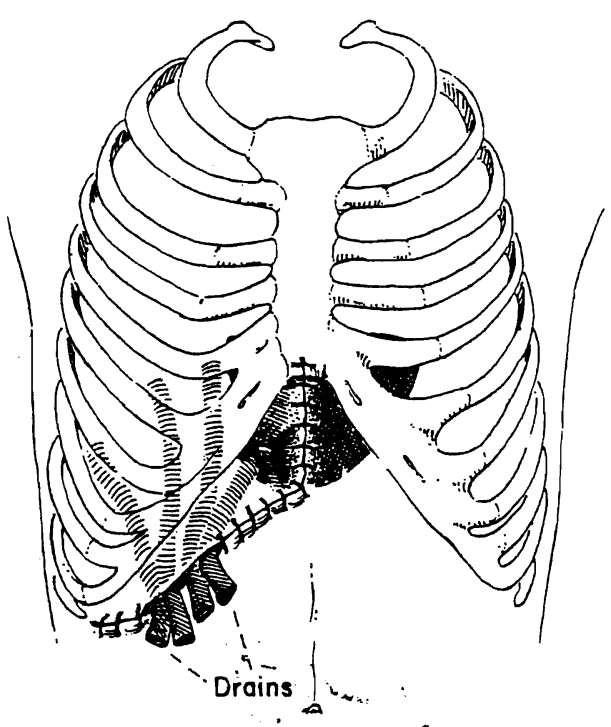

Fig. 9

FIG. 8
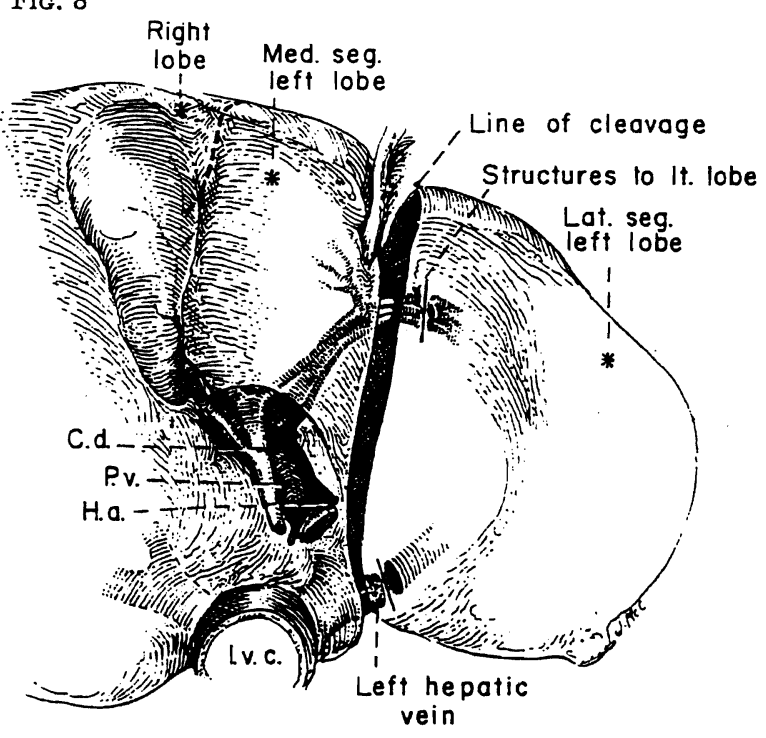

Fig. 10

Fig. 8. Liver transection nearly completed along exact line of color change demarcated by viable and cyanotic liver tissue. Intersegmental veins are left attached to the lateral segment if possible. The last major structure to be encountered is the middle hepatic vein.

FrG. 9. Wound drainage after trisegmentectomy. Usually, either the medial or lateral portion of the wound is left open for 3 or 4 inches.

Fig. 10. Site of ligation of portal structures for lateral segmentectomy. The dissection is kept to the left of the umbilical fissure to prevent injury to the structures feeding back from the fissure to the medial segment.

Lin's adaptation of a special clamp originally described by Storm and Longmire (18) to control bleeding from the raw surface. However, Lin's techniques have not been adaptable to the performance of the more extensive trisegmentectomy which is often required to remove a tumor with adequate margins. They probably do not permit even a complete true lobectomy. There were no trisegmentectomies in Lin's series (8). Because of this important limitation, an indisposition to teach a technique so much at variance with surgical principles of extirpation applicable to other organs 
and satisfaction with more traditional control techniques, we have not used Lin's methods.

Recently, Fortner and his associates (3) described chilling of the whole liver by infusion techniques developed for liver transplantation. After this preliminary step, the diseased portion of the organ was removed in a bloodless field. A mortality rate of 10 per cent was reported in 29 operations of which at least 26 were lesser procedures than trisegmentectomy. The results in our experience have indicated that even the radical trisegmentectomy can be safely achieved with a far less complicated approach. Since the same is doubtless true for lesser procedures, such as true hepatic lobectomy or segmentectomy, we have perceived no justification for this approach.

\section{SUMMARY}

Trisegmentectomy, extended right hepatic lobectomy, is the removal of the true right lobe of the liver in continuity with most or all of the medial segment of the left lobe. Some important features of the operation have not been well described previously. To perform trisegmentectomy safely, a fusion of liver tissue covering the umbilical fissure at the level of the falciform ligament must first be split open in many patients. The left branches of the portal triad structures are mobilized from the undersurface of the liver nearly to but not into the umbilical fissure. The blood supply and duct drainage of the medial segment originate within the umbilical fissure and feed back toward the right side buried in liver substance. They are found with blunt dissection just to the right of the falciform ligament, encircled and ligated. Failure to appreciate this switch back anatomic arrangement may lead to injury of the blood supply or biliary drainage of the residual lateral segment. Parenthetically, the mirror image operation of lateral segmentectomy could result in devascularization of the medial segment if dissection and ligation were performed within the umbilical fissure instead of well to the left of this landmark.

In most trisegmentectomies, the left portion of the caudate lobe is not removed. This small piece of tissue is interposed between the lateral segment and the inferior vena cava into which it drains by small tributaries. If the left portion of the caudate lobe is to be excised, it is necessary to ligate the last two posteriorly running branches before the main left trunks of the portal triad structures reach the umbilical fissure. Once this step is taken and if the caudate removal is completed, the remaining lateral segment usually has only one remaining outflow, that of the left hepatic vein.
The other principles of trisegmentectomy are the same as with less radical subtotal hepatic resection. These include vascular suture closure of the main outflow veins, avoidance of parasegmental planes that leave behind a strip of devitalized tissue, preservation of intersegmental or interlobar veins, omission of techniques that sew shut or otherwise cover the raw surface of the remnant and provision of adequate drainage of dead space. After trisegmentectomy and also after true lobectomy, this last objective is usually met by leaving part of the operative incision open.

Using these guidelines, there has been no mortality with 27 hepatic resections carried out since 1963, including 14 trisegmentectomies.

\section{REFERENCES}

1. BRUNSCHWTG, A. The surgery of bepatic neoplasms with special reference to right and left lobectomies. XVI Congress de la Societe Internationale de Chirurgie, Copenhagen. Pp. 1122-1132. Brussels: Imprimerie Medicale et Scientifique, 1955.

2. Counnaud, C. Le Foie. Etudes Anatomiques et Chirurgicales. Paris: Masson, 1957.

3. Fortner, J. G., SHIU, M. H., KINNE, D. W., and others. Major hepatic resection using vascular isolation and hypothermic perfusion. Ann. Surg., 1974, 180: 644.

4. GoldsMTt, N. A., and WoOdBurNe, R. T. The surgical anatomy pertaining to liver resection. Surg. Gynecol. Obstet., 1957, 105: 310.

5. Henley, J. E., JR. Clinical anatomic aspects of radical hepatic surgery. J. Int. Coll. Surg., 1954, 22: 542.

6. HEAlEY, J. E., JR., and SCHroy, P. C. Anatomy of the biliary ducts within the human liver. Arch. Surg., 1953, 66: 599 .

7. HJORTsjö, C. H. The topography of the intrahepatic duct systems. Acta Anat., 1950, 11: 599.

8. LIN, T-Y. Results in 107 hepatic lobectomies with a preliminary report on the use of a clamp to reduce blood loss. Ann. Surg., 1973, 177: 413.

9. LiN, T-Y., CHEN, K. M., and Lru, T. K. Total right hepatic lobectomy for primary hepatoma. Surgery, 1960, 40: 1048 .

10. Longurre, W. R., Jr., Trout, H. H., III, Greenfield, J., and Tompxins, R. K. Elective hepatic surgery. Ann. Surg., 1974, 179: 712.

11. Lortat-Jacob, J. L., and Robert, H. G. Hepatectomie droite reglee. Presse Med., 1952, 60: 549.

12. McBride, C. M., and Wallace, S. Cancer of the right lobe of the liver. Arch. Surg., 1972, 105: 289.

13. MCIndoe, A. H., and CounselleR, V. S. The bilaterality of the liver. Arch. Surg., 1927, 15: 589.

14. Milier, T. R. Neoplasms of the liver. In: Abdominal Operations. Edited by R. Maingot. Vol. II, pp. 12261246. New York: Appleton-Century-Grofts, 1974.

15. PACx, G. T., Miller, T. R., and Brasfield, R. D. Total right hepatic lobectomy for cancer of the gallbladder. Ann. Surg., 1955, 142: 6.

16. PACK, G. T., and Molander, D. W. Metabolism before and after hepatic lobectomy for cancer. Arch. Surg., 1960, 80: 685 .

17. Quattrebaum, J. K. Massive resection of the liver. Ann. Surg., 1953, 137: 787.

18. Storm, F. K., and Longure, W. P., JR. A simplified clamp for hepatic resection. Surg. Gynecol. Obstet., 1971, 133: 103 .

19. WANGensteen, O. H. Cancer of the esophagus and stomach. In: Proceedings 3rd Natl. Cancer Conference. Pp. 94-97. Philadelphia: J. B. Lippincott Co., 1951. 